\title{
Multimodal Nonlinear Optical Microscopy for Simultaneous 3-D Label-Free and Immunofluorescence Imaging of Biological Samples
}

\author{
Joo Hyun Park ${ }^{1,2}$, Eun-Soo Lee ${ }^{2}$ Jae Yong Lee ${ }^{1,3}$, Eun Seong Lee ${ }^{4}$, Tae Geol Lee ${ }^{1,2}$, \\ Se-Hwa Kim ${ }^{1,2 *}$, and Sang-Won Lee ${ }^{1,2 \dagger}$ \\ ${ }^{I}$ Department of Nano and Bio Surface Science, Korea University of Science and Technology, \\ 217 Gajeong-ro, Yusoeng-gu, Daejeon 305-350, Korea \\ ${ }^{2}$ Center for Nanosafety Metrology, Korea Research Institute of Standards and Science, \\ 267 Gajeong-ro, Yuseong-gu, Daejeon 305-340, Korea \\ ${ }^{3}$ Center for Length, Korea Research Institute of Standards and Science, \\ 267 Gajeong-ro, Yuseong-gu, Daejeon 305-340, Korea \\ ${ }^{4}$ Center for Nanometrology, Korea Research Institute of Standards and Science, \\ 267 Gajeong-ro, Yuseong-gu, Daejeon 305-340, Korea
}

(Received July 2, 2014 : revised September 23, 2014 : accepted September 23, 2014)

\begin{abstract}
In this study, we demonstrated multimodal nonlinear optical (NLO) microscopy integrated simultaneously with two-photon excitation fluorescence (TPEF), second-harmonic generation (SHG), and coherent anti-Stokes Raman scattering (CARS) in order to obtain targeted cellular and label-free images in an immunofluorescence assay of the atherosclerotic aorta from apolipoprotein E-deficient mice. The multimodal NLO microscope used two laser systems: picosecond (ps) and femtosecond (fs) pulsed lasers. A pair of ps-pulsed lights served for CARS $(817 \mathrm{~nm}$ and $1064 \mathrm{~nm})$ and SHG $(817 \mathrm{~nm})$ images; light from the fs-pulsed laser with the center wavelength of $720 \mathrm{~nm}$ was incident into the sample to obtain autofluorescence and targeted molecular TPEF images for high efficiency of fluorescence intensity without cross-talk. For multicolor-targeted TPEF imaging, we stained smooth-muscle cells and macrophages with fluorescent dyes (Alexa Fluor 350 and Alexa Fluor 594) for an immunofluorescence assay. Each depth-sectioned image consisted of $512 \times 512$ pixels with a field of view of $250 \times 250 \mu^{2}$, a lateral resolution of $0.4 \mu \mathrm{m}$, and an axial resolution of $1.3 \mu \mathrm{m}$. We obtained composite multicolor images with conventional label-free NLO images and targeted TPEF images in atherosclerotic-plaque samples. Multicolor 3-D imaging of atherosclerotic-plaque structural and functional composition will be helpful for understanding the pathogenesis of cardiovascular disease.
\end{abstract}

Keywords : Nonlinear optical microscopy, Multimodal, Immunofluorescence assay

OCIS codes : (170.3880) Medical and biological imaging; (170.0180) Microscopy; (180.4315) Nonlinear microscopy

\section{INTRODUCTION}

Nonlinear optical (NLO) microscopy is a powerful imaging technique because it can provide subcellular-scale 3-D images of biological samples. NLO microscopy provides high spatial resolution and deep penetration (several hundreds of micrometers) using near infrared (NIR) wavelength excitations compared to conventional confocal-fluorescence microscopy. In addition, NLO microscopy is sensitive to specific molecules and structures [1-6]. Recently, multimodal NLO microscopy has been extended to include two-photon excitation fluorescence (TPEF), second-harmonic generation (SHG), and coherence anti-Stokes Raman scattering (CARS) imaging on the same platform [7-9]. Multimodal NLO microscopy allows simultaneous visualization of different endogenous and exogenous structures and specific molecules in complex tissue samples. Most studies using multimodal NLO microscopy have been directed toward label-free microscopic imaging because of the high

\footnotetext{
*Corresponding authors: *shkim@kriss.re.kr and swlee76@kriss.re.kr

Color versions of one or more of the figures in this paper are available online.
} 
sensitivity and specificity for major extracellular molecules [10-12]. In previous studies of multimodal NLO microscopy, TPEF was used to specifically image extracellular elastin fibers without staining. SHG and CARS can be used to obtain microscopic images of collagen fibrils and lipid-rich structures (or extracellular lipid droplets), respectively, without any staining.

However, although there are advantages to label-free imaging, targeted imaging techniques using fluorescent protein or dye are still widely used in fields such as biology, biophysics, biochemistry, and neuroscience because multicolor, targeted images can provide a range of diagnostics simultaneously - tracking of cell anatomy, movement, lineage, visualization of specific molecules, and recording of activity and signaling events [13]. Recently, multicolor imaging techniques in TPEF microscopy have been studied [14-16]. Kawano et al. [14] and Tillo et al. [15] have proposed the combination of two fluorescent proteins having a single excitation wavelength and well-separated emission spectra; various fluorescent proteins and dyes have high two-photon absorption cross-sections between $720 \mathrm{~nm}$ and $860 \mathrm{~nm}$ and may emit fluorescence at each emission wavelength region corresponding to single-photon excitation. Mahou et al. has demonstrated multicolor two-photon tissue imaging using three fluorescent chromophores with distinct absorption spectra [16]. They used the fundamental wavelength $\left(\lambda_{1}\right)$ from the laser, another wavelength $\left(\lambda_{2}\right)$ from an optical parametric oscillator (OPO), and the "virtual" wavelength, $\lambda_{3}=2 /\left(1 / \lambda_{1}+1 / \lambda_{2}\right)$. The phenomenon of excitation by virtual wavelength is termed "two-color two-photon excitation" [16].

In previous multimodal NLO microscopy papers, a single picosecond (ps) pulsed laser system, a single femtosecond (fs) pulsed laser system with a photonic crystal fiber (PCF), or multi-laser systems with a flip mirror for switching have been used [7-12]. However, in this study, we constructed a multimodal NLO microscope using lights that were combined of an fs-pulsed laser and a ps-pulsed laser incident into a sample, simultaneously. Generally, for label-free imaging, the fs-pulsed laser has been used to generate SHG and TPEF, simultaneously, because the intensities of SHG and TPEF at fs-pulsed light are higher than those at ps-pulsed light [17]. However, in this study, we used the ps-pulsed laser for SHG and the fs-pulsed laser for TPEF when the pulse duration and the wavelength were considered to obtain simultaneously multimodal and multicolor images without any laser parameter changing. As a biological sample, we used the atherosclerotic aorta from an apolipoprotein E-deficient $\left(\mathrm{ApoE}^{-/}\right)$mouse with the goal of obtaining simultaneous label-free and targeted molecular images. Recently, atherosclerosis has been studied as one of the medical applications of multimodal NLO microscopy [18-22]. Atherosclerosis is a chronic, progressive arterial disease associated with lipid deposition and chronic inflammation $[23,24]$. Atherosclerotic lesions have biomolecules associated with endothelial cells, extracellular lipid droplets, lipid-rich cells, low-density lipoprotein aggregates, collagen, and elastin.
TPEF, SHG, and CARS have been demonstrated for imaging extracellular elastin, type-I collagen fibrils, and lipid-rich molecules, respectively, for non-stained atherosclerotic plaque [18-22]. However, analysis of label-free visualizations of elastin, collagen, and lipids are limited by complicated morphological or chemical changes in atherosclerotic progress. Therefore, NLO microscopy is required in addition for complementary information such as targeted molecular and cellular imaging, in order to understand the pathogenesis of cardiovascular disease. Immunoassay, as the gold standard, can provide identification of the cellular constituents in atherosclerosis studies of inflammatory disease involving several cell types, including macrophages (MФs) and smooth muscle cells (SMCs) [25-27]. Immunohistochemistry (IHC) and immunofluorescence (IF) have been commonly used by pathologists in diagnostic evaluations in order to identify specific cells, organs, and structures [28, 29]. A limitation of these two methods is that they must be performed on thin slices of atherosclerosis samples. Although confocal laser scanning fluorescence microscopy has routinely been used for 3-D studies of samples, it has a limited penetration depth and the resolution deteriorates with depth [30, 31]. To avoid these problems, TPEF microscopy can be applied to IF assay. We used fluorescent dyes - Alexa Fluor 350 and Alexa Fluor 594 - in order to obtain multicolor, labeled TPEF images; we varied our excitation wavelength over the range 720-800 $\mathrm{nm}$ in order to determine the wavelength dependence of the two-photon absorption crosssections. By using multimodal NLO microscopy, information on plaque composition and structural organization has been obtained from multicolor label-free images; multicolor targeted images have been used to identify specific molecular and cellular information.

\section{EXPERIMENTAL SETUP}

The schematic of the multimodal NLO microscope is shown in Fig. 1. In this study, we used two separate laser systems - a ps-pulsed laser (picoEmerald, APE GmbH, Berlin, Germany) and a fs-pulsed Ti:sapphire laser (Chameleon Vision-S, Coherent Inc., Santa Clara, CA) - with orthogonal polarizations. The ps-pulsed laser for CARS imaging was integrated with an OPO and a frequency-doubled Nd:YVO laser that delivered 7-ps pulses with a repetition rate of 80 $\mathrm{MHz}$ at $1064 \mathrm{~nm}$. The fundamental 1064-nm wavelength laser beam was used as the Stokes beam $\left(\omega_{s}\right)$. When a frequency-doubled beam $(532 \mathrm{~nm})$ from the $\mathrm{Nd}$ :YVO laser was incident into a temperature-tuned lithium triborate (LBO) crystal in the OPO, temporally and spatially overlapped signal and idler outputs with 6-7-ps pulse trains were generated, which were tunable within $720-990 \mathrm{~nm}$ and $1150-2030 \mathrm{~nm}$ ranges, respectively. The signal beam, used as a pump beam $\left(\omega_{p}\right)$, was set to $817 \mathrm{~nm}$ in order to obtain CARS images of lipids (Raman shift of $2845 \mathrm{~cm}^{-1}$ ). Two beams at $817 \mathrm{~nm}$ and $1064 \mathrm{~nm}$ were also temporally 

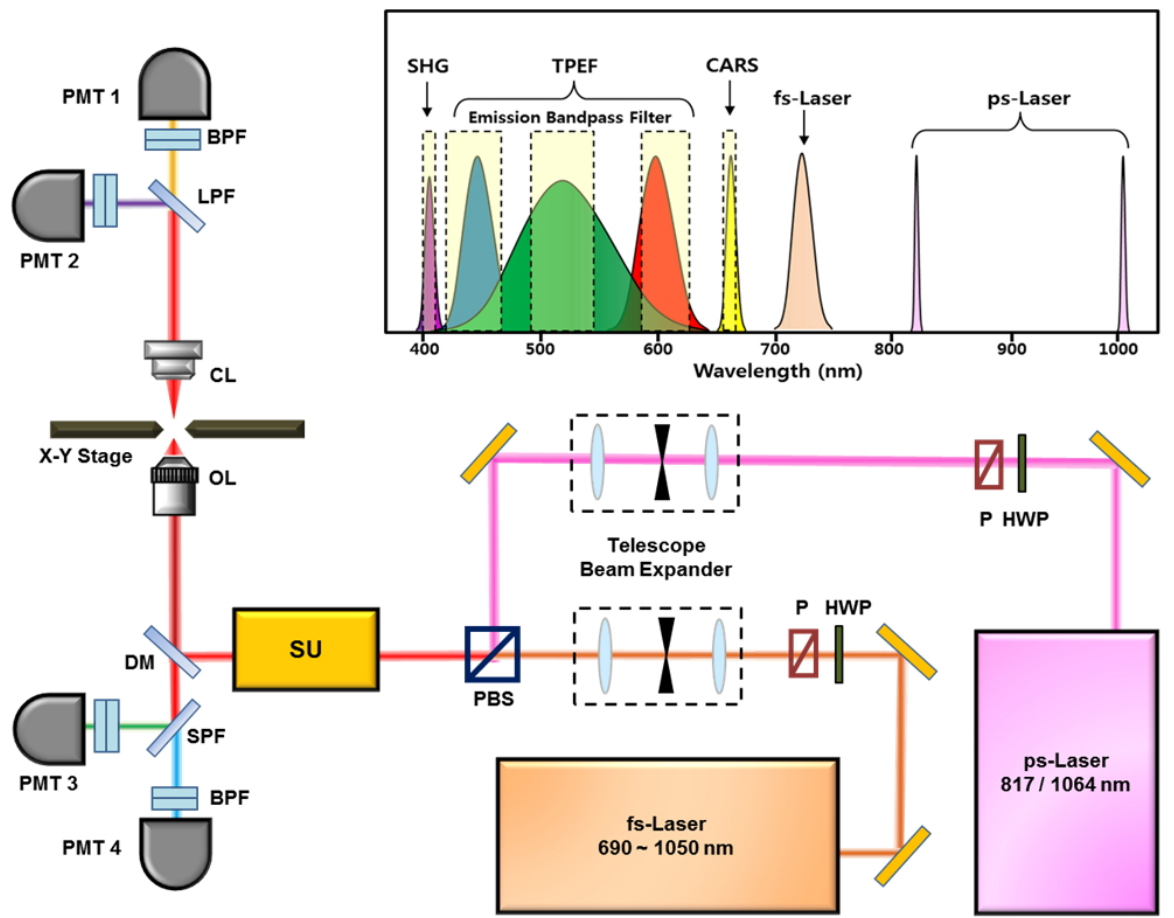

FIG. 1. Schematic of multimodal nonlinear optical (NLO) microscopy. The inset shows a wavelength spectrum of the laser sources and sample emissions. HWP: half-wave plate, P: polarizer, PBS: polarization beamsplitter, DM: dichroic mirror, OL: objective lens, CL: collection lens, SPF: short-pass filter, LPF: long-pass filter, BPF: bandpass filter, SU: scanning unit, and PMT: photomultiplier tube.

and spatially overlapped in the integrated ps-pulsed laser. The center wavelength of the fs-pulsed Ti:sapphire laser was tunable from 690-1050 nm with a pulse width of 75 fs and a repetition rate of $80 \mathrm{MHz}$. As shown in Fig. 1, the combination of an achromatic half-wave plate and a Glan-Thompson polarizer was used to control the attenuation and polarization of each of the laser beams. The Stokes beam and pump beam from the ps-pulsed laser were rotated to $s$-polarization (vertically polarized). The beam from the fs-pulsed laser was set to $p$-polarization (horizontally polarized). Two $s$-polarized beams and one $p$-polarized beam were combined by a polarized beamsplitter (PBS). The beam diameter and divergence of each laser beam was adjusted by a telescope-beam expander placed in each beam path. All beams were delivered into a modified, inverted-type multiphoton microscope (FV1000MPE + IX81, Olympus, Japan). Some of the optics in the microscope were customized in order to ensure sufficient optical throughput at near-IR wavelengths. A $60 \times$ water-immersion objective lens with $\mathrm{NA}=1.2$ (UPlanSApo/UIS2, Olympus, Japan) was used to focus all of the laser beams into the specimen.

The CARS and SHG signal from the specimen were collected using a condenser lens (IX2-LWUCDA2, Olympus, Japan) with NA=0.55 and a $27-\mathrm{mm}$ working distance in the forward direction and two external photomultiplier tubes (PMT1 and PMT2 in Fig. 1). To separate CARS and SHG signals, we used a dichroic mirror and placed 10-nm wide optical bandpass filters with center wavelengths of
$660 \mathrm{~nm}$ and $405 \mathrm{~nm}$, respectively, on the front of each PMT. The TPEF signals from the specimen were collected by the same objective in the backward (epi-) direction and separated by a dichroic mirror. The autofluorescent emission wavelength of elastin had the maximum efficiency at 520 $\mathrm{nm}$ (green). Therefore, the emission filters of blue (420-460 $\mathrm{nm})$, green $(490-540 \mathrm{~nm})$, and red $(610-650 \mathrm{~nm})$ were used in order to avoid crosstalk among the fluorochromes. Images were displayed and analyzed using FluoView software (FV10-ASW, Olympus Corp., Japan). Our multimodal NLO microscope could acquire 3-D en-face images having a maximum field of view of $250 \times 250 \mu \mathrm{m}^{2}$ with spatial resolutions of $0.4 \mu \mathrm{m}$ in the lateral $(x-y)$ plane and $1.3 \mu \mathrm{m}$ along the axial direction $(z)$. Each depth-sectioned image consisting of $512 \times 512$ pixels could be obtained at a frame rate of $1.2 \mathrm{fps}$.

\section{ANIMAL MODEL AND TISSUE PREPARATION}

Male $\mathrm{ApoE}^{-/}$mice were purchased from Jackson Laboratory (Bar Harbor, ME). The ApoE ${ }^{-/}$mice were fed a high-fat diet (\#D12079B, Research Diets, New Brunswick, NJ) for 20-30 weeks and maintained under pathogen-free conditions at the Korea Research Institute of Bioscience and Biotechnology. All animal studies conformed to the tenets of the Institutional Animal Care and Use Committee of the 
Korea Research Institute of Bioscience and Biotechnology. Mice were euthanized with $\mathrm{CO}_{2}$ inhalation and then the vasculature was flushed with phosphate-buffered saline for $5 \mathrm{~min}$. The aorta section from aortic root to thoracic aorta was isolated as described in our previous study [32]. After removing the perivascular connective tissue, a longitudinally opened descending aorta was mounted lumen-side down on a coverglass-bottom chamber, covered with a coverslip.

Cross-sectional and longitudinal-sectioned aortae of the mice were fixed with $4 \%$ paraformaldehyde for $10 \mathrm{~min}$. and washed with phosphate-buffered saline (PBS) containing $0.1 \%$ bovine serum albumin (BSA). The fixed aortae were permeabilized with PBS containing $0.1 \%$ BSA, $0.5 \%$ Triton $\mathrm{X}-100$, and $10 \%$ goat serum for $10 \mathrm{~min}$. Then, specimens were incubated with anti-CD68 (1:100, Abcam; UK), anti-smooth muscle a-actin (1:200, Sigma, St. Louis, MO) antibodies at $4^{\circ} \mathrm{C}$ overnight. The anti-smooth muscle a -actin is used to identify SMCs and the anti-CD68 antibody is used to target MФs. After washing with PBS, specimens were incubated with secondary antibody-conjugated Alexa Fluor 350 and Alexa Fluor 594 (Molecular Probe, Eugene, OR) for 1 hour at room temperature and then loaded into a Lab-Tek II two-well chambered coverglass (Nalge Nunc International, Rochester NY) in order to obtain labeled TPEF images.

\section{RESULTS AND DISCUSSION}

First, we carried out targeted molecular imaging in order to demonstrate the capability of our multimodal NLO microscopy and to characterize the TPEF images of atherosclerotic plaques. $\mathrm{M} \Phi$ and SMC have been studied as important markers for the characterization of atherosclerotic plaques. Therefore, we obtained TPEF images of M $\Phi$ and SMC using traditional histological IF techniques in multimodal NLO microscopy. Before obtaining the TPEF images, we prepared the atherosclerotic aortae of the mice with slices $10 \mu \mathrm{m}$ thick in the cross-sectional direction. Because the Alexa Fluor 350 and Alexa Fluor 594 used in this study have different two-photon absorption cross-sections [33], it is essential to select a suitable excitation wavelength for the two fluorophores. In order to determine a wavelength with high efficiency, we scanned the wavelength of the fs-pulsed laser from 720 to $800 \mathrm{~nm}$. Figures 2(a) to (c) are TPEF images with an excitation wavelength of 800 $\mathrm{nm}$. The TPEF images of Fig. 2(d) to (f) were obtained at $720 \mathrm{~nm}$. Figures 2(a) and (d) show the typical label-free (autofluorescence) image of the elastin as a major component of the internal elastic lamina (IEL). We could not determine a difference between $800 \mathrm{~nm}$ - and $720 \mathrm{~nm}$-excited autofluorescence images. However, SMCs (blue) stained with Alexa 350 could not be observed with $800-\mathrm{nm}$ excitation. Excitation with $800 \mathrm{~nm}$ significantly reduced fluorescence intensities in the TPEF images because blue-color fluorophores such as DAPI, SYTO 40, and Alexa 350 have a maximum excitation-wavelength in the vicinity of $700 \mathrm{~nm}$. On the contrary, the Alexa 594 has slightly lower emission quantum efficiency at $720 \mathrm{~nm}$ than at $800 \mathrm{~nm}$. Nevertheless, the difference of emission quantum efficiency between $720 \mathrm{~nm}$ and $800 \mathrm{~nm}$ did not significantly affect the ability to obtain images as shown in Fig. 2(c) and (f). Therefore, we found that an excitation wavelength of 720 $\mathrm{nm}$ was suitable for obtaining simultaneously two targeted images from samples stained with Alexa 350 and Alex 594. TPEF signal intensity is inversely proportional to the pulse width of the laser [17]. The pulse duration of the fs-pulsed laser became longer because light passed through several optical components such as lenses, PBS, and the objective lens in our system. Therefore, we adjusted the negative dispersion value of the pre-compensator integrated within the laser in order to maximize the TPEF signal.

Figures $2(\mathrm{~g})$ and $(\mathrm{h})$ show SHG and CARS images corresponding to collagen fibrils and lipids, respectively. The collagen fibrils were predominantly distributed in adventitia imaged by SHG using the pump beam $(817 \mathrm{~nm})$

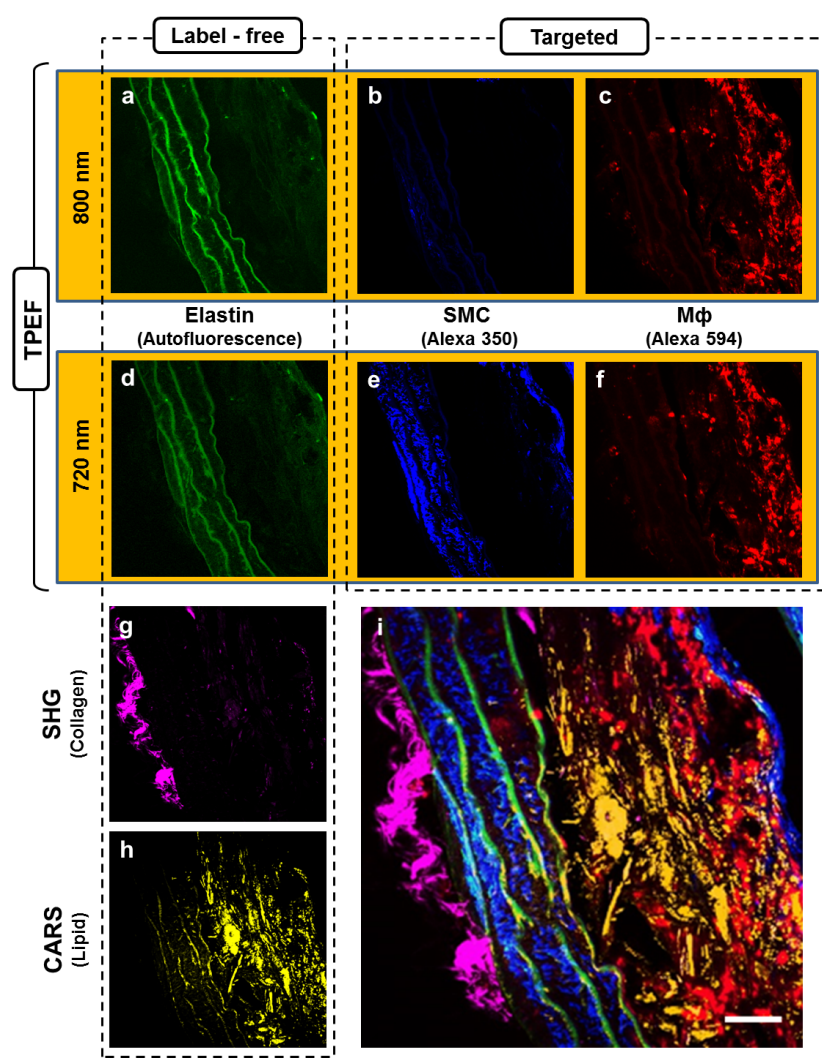

FIG 2. Multimodal nonlinear microscopic images of atherosclerotic plaques in the cross-sectional direction. (a)-(c) TPEF images at $800 \mathrm{~nm}$ of the fs-pulsed laser, (d)-(f) TPEF images at $720 \mathrm{~nm}$ of the fs-pulsed laser, (g) SHG image at $817 \mathrm{~nm}$ of the ps-pulsed laser, (h) CARS image at $817 \mathrm{~nm}$ and $1064 \mathrm{~nm}$ of the ps-pulsed laser, and (i) merged image of (d)-(h). (a) and (d) are autofluorescence TPEF images of elastin. (b) and (e) are TPEF images of smooth muscle cells (SMCs) Stained with Alexa 350. Finally, (c) and (f) are TPEF images of macrophages (MФs) stained with Alexa 594. The scale bar in (i) is $20 \mu \mathrm{m}$. 
of the ps-pulsed laser and the optical bandpass filter with $405 \pm 10 \mathrm{~nm}$. SHG signals from the collagen fibrils could be generated from excitation at $720 \mathrm{~nm}$ by the fs-pulsed laser because the ps- and fs-pulsed lasers were simultaneously incident into the sample. However, the SHG signal could not be detected despite the use of PMTs having a spectral detection range of 196-900 $\mathrm{nm}$ because the anti-reflection coating of the condenser lens at forward direction was optimized at visible wavelength. In addition, the intensity of the SHG signal is sensitive to variations in the incident wavelength. The intensity of the SHG signal in collagen fibrils at longer wavelengths is higher than at shorter wavelengths [34]. Generally, it is well known that the pulse width of the pulsed laser also affects SHG signals. As the laser pulse width is shortened, the generated SHG signals become stronger [17]. However, we found that the SHG signals were more strongly dependent on the center wavelength of the excitation than on the pulse width. CARS imaging reveals significant lipid accumulation. The strong CARS signal from the foam cells specifically shows the distribution of lipid structures.

We could obtain two color-targeted TPEF images and one label-free TPEF image, which could be clearly distinguished without fluorophore crosstalk because of the use of a set of bandpass filters as shown in the inset graph of Fig. 1. However, we could simultaneously obtain only four images - two targeted TPEF images, an SHG image, and a CARS image - because our multimodal NLO microscope had only four PMTs. After acquiring four images, we changed the optical filter in one of PMTs to the backward direction and obtained the autofluorescence TPEF image of elastin. Finally, we could obtain the merged five-color image shown in Fig. 2 (i). As shown in Fig. 2 (i), the IEL were clearly identified in the media, SMCs were densely packed between IEL layers, and MФs were rarely and randomly distributed on plaque lesions. This composite image verified that multimodal NLO microscopy offers simultaneous label-free and targeted molecular imaging. Our microscope system including FluoView software can be augmented with two additional PMTs in the backward direction. With the addition of two PMTs in combination with well-separated optical-bandpass filters and sample fluorophores, the microscope would be able to obtain simultaneous six-color label-free and targeted molecular images.

Figures 3 and Figure 4 are multi-color images of an atherosclerotic aorta, which was cut in the longitudinal direction as shown in Fig. 3(a). In order to visualize the 3-D structural information in atherosclerotic plaques, multi-color images were taken at successive $1-\mu \mathrm{m}$ steps in the axial (z) direction, from adventitia to lumen. Figure 3(b) shows stacked multi-color images with axial intervals of $10 \mu \mathrm{m}$. Depending on the depth of the atherosclerotic aorta, the curved collagen fibrils (magenta) located in adventitia were visualized at $0-5 \mu \mathrm{m}$ in depth, as shown in Fig. 3(b). The elastin (green) in the IEL layer existed at a depth of approximately 10-30 $\mu \mathrm{m}$. In addition, we could observe SMCs (blue) at 10-30 $\mu \mathrm{m}$. The distribution of the SMCs and $\mathrm{M} \Phi \mathrm{s}$ (red) were similar in the image in the cross-sectional direction, as shown in Fig. 2. The lipid-laden foam cells (yellow) are first visualized at a depth of $40 \mu \mathrm{m}$ from the adventitia. The foam cells have mainly originated from $\mathrm{M}$ Фs. However, occasionally several foam cells exhibiting morphological characteristics of SMC are observed [35]. Figure 3(c) is the 3-D image consisting of 81 multi-color NLO images with $z$-sectioning at $1-\mu \mathrm{m}$ intervals.

Figure 4 shows targeted cellular images of SMCs

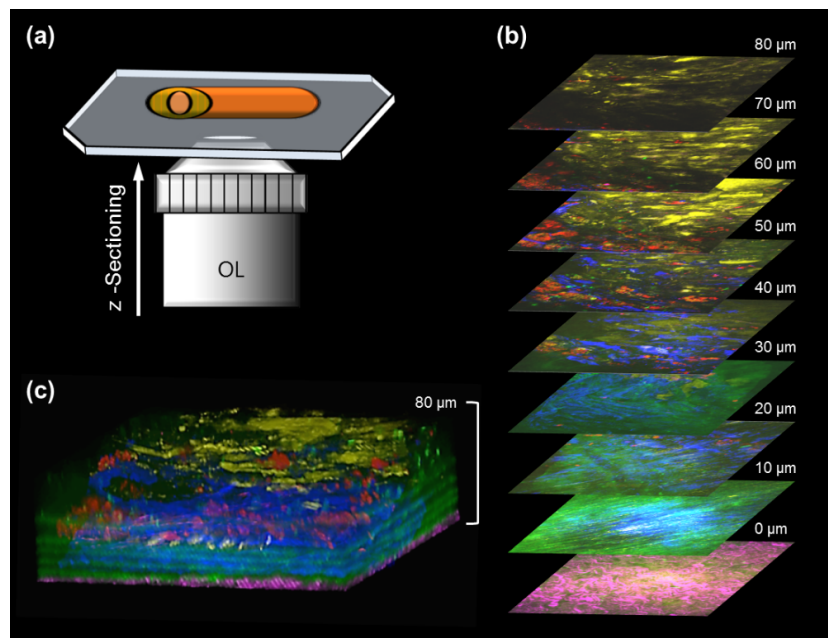

FIG. 3. Multi-color label-free and targeted molecular images of the atherosclerotic aorta in the depth direction. (a) Schematic diagram of the segmentation of the aorta in the image-acquisition direction, (b) multi-color images at intervals of $10 \mu \mathrm{m}$, and (c) the reconstructed 3-D image consisting of 81 multi-color NLO images with $1-\mu \mathrm{m} z$-sectioning.
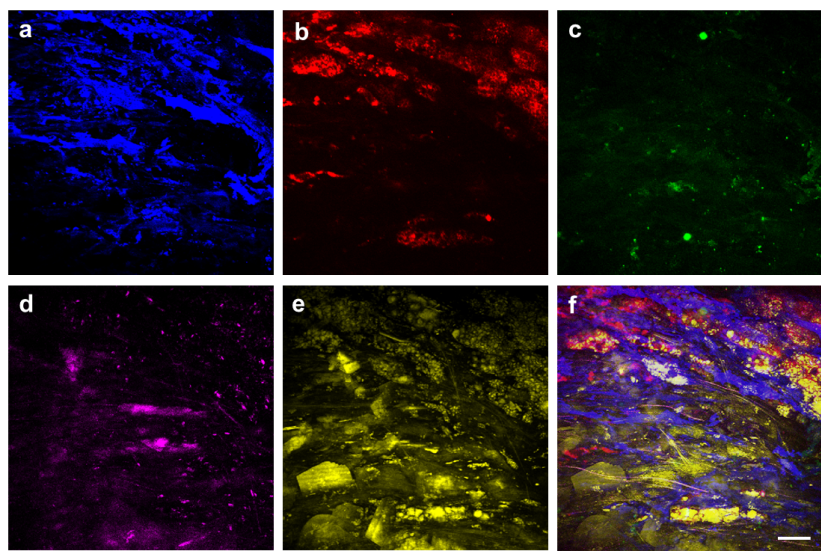

FIG 4. Multi-color label-free and targeted molecular images of the atherosclerotic aorta in longitudinal direction. (a) TPEF image of SMCs stained with Alexa 350, (b) TPEF image of M $\Phi$ s stained with Alexa 594, (c) autofluorescence TPEF image of elastin, (d) SHG image of collagen fibril, (e) CARS image of lipid droplets and crystals, and (f) merged image of (a)-(e). The scale bar in (f) is $20 \mu \mathrm{m}$. 
and MФs (b) using TPEF, along with representative multimodal NLO images of the elastin (c), the collagen (d), and the lipids (e) of the atherosclerotic plaques in another area of the specimen at the depth of approximately $50 \mu \mathrm{m}$ from adventitia. SMCs were widely distributed between layers of IEL, similar to Fig. 3(b). In Fig. 4(c), the elastin could not be detected by TPEF because the elastin did not exist in this depth. As shown in Fig. 3(b), the elastin existed at a depth of approximately 10-30 $\mu \mathrm{m}$. In addition, collagen fibrils were invisible in Fig. 4(d) because collagen fibrils located at $0-5 \mu \mathrm{m}$ depth from adventitia were visualized. $\mathrm{M} \Phi$ s were clearly visible and were abundant underneath the lipid core in the form of macrophage-derived foam cells, as shown in Fig. 4(f). The composite image in Fig. 4(f) could be used to characterize the distribution of SMCs and $M \Phi s$ as well as the specific composition of extracellular matrix (ECM) containing the IEL layer, the collagen structure, and the lipid accumulation. The multicolor 3-D image can reveal the interaction between the cell and the ECM as well as details of morphological and structural events. This multicolor 3-D imaging provides novel opportunities for mechanistic understanding of disease progressions. Advantages of our scheme are that a multicolor 3-D image can be acquired coincidentally by integrating independent nonlinear processes, and the single wavelength excitation of the multiple chromophores provides faster acquisition time than linear microscopy such as confocal-fluorescence microscopy. Additionally, this approach can be applied to a variety of organs imaging with the other target molecules and fluorophore combination in biomedical research fields. In this study, we applied targeted TPEF images to the SMCs and M $\Phi$ s, which are closely related in inflammation and key roles in the pathogenesis of atherosclerosis. [36-38]. However, the use of various biomarkers in multimodal and multicolor NLO microscopy will be helpful for understanding the pathogenesis of cardiovascular disease [39].

\section{CONCLUSION}

We visualized key aspects of atherosclerotic processes - such as SMCs, MФs, foam cells, and the ECM - using multimodal NLO microscopy in a labeled and label-free manner. We obtained TPEF, SHG, and CARS images of elastin, collagen fibrils, and lipids, respectively, without staining. Additionally, we used fluorescent dyes to obtain multicolor TPEF images of the MФs and SMCs in the atherosclerotic aortae of $\mathrm{ApoE}^{-/-}$mice. The combination of label-free and targeted molecular NLO imaging modalities is particularly advantageous for simultaneously identifying the location and properties of the cellular components and morphological structures in atherosclerotic plaques. In particular, because of the larger penetration depth compared with conventional immunofluorescence assays, the TPEF images of the selective and targeted molecules have the potential to expand the understanding of the complex interplay between the targeted agent and the ECM. Our approach will be useful in various biomedical fields such as biology, biophysics, biochemistry, and neuroscience as well as understanding the pathogenesis of cardiovascular disease.

\section{ACKNOWLEDGMENT}

Joo Hyun Park and Eun-Soo Lee contributed equally to this work as co-first authors. This work was supported by the "Development of Nanobio Convergence and Nanomaterial Safety Technology Program (GP2014-0019)" from the Korea Research Institute of Standards and Science; the "Bio-signal Analysis Technology Program (2006-2005082)" and "Pioneer Research Center Program (2012-0009541)" through the National Research Foundation (NRF) funded of Korea by the Ministry of Science, ICT \& Future Planning.

\section{REFERENCES}

1. W. Denk, J. H. Strickler, and W. W. Webb, "Two-photon laser scanning fluorescence microscopy," Science 248, 73-76 (1990).

2. C. Buehler, K. H. Kim, C. Y. Dong, B. R. Masters, and P. T. So, "Innovations in two-photon deep tissue microscopy," IEEE Eng. Med. Biol. Mag. 18, 23-30 (1999).

3. I. Freund, M. Deutsch, and A. Sprecher, "Connective tissue polarity. Optical second-harmonic microscopy, crossed-beam summation, and small-angle scattering in rat-tail tendon," Biophys. J. 50, 693-712 (1986).

4. P. Campagnola, "Second harmonic generation imaging microscopy: Applications to diseases diagnostics," Anal. Chem. 83, 3224-3231 (2011).

5. R. F. Begley, A. B. Harvey, and R. L. Byer, "Coherent antiStokes Raman spectroscopy," Appl. Phys. Lett. 25, 387-390 (1974).

6. M. D. Duncan, J. Reintjes, and T. J. Manuccia, "Scanning coherent anti-Stokes Raman microscope," Opt. Lett. 7, 350-352 (1982).

7. T. B. Huff, Y. Shi, Y. Fu, H. Wang, and J. X. Cheng, "Multimodal nonlinear optical microscopy and applications to central nervous system imaging," IEEE J. Select. Topics Quantum Electron. 14, 4-9 (2008).

8. H. Chen, H. Wang, M. N. Slipchenko, Y. Jung, Y. Shi, J. Zhu, K. K. Buhman, and J. X. Cheng, "A multimodal platform for nonlinear optical microscopy and microspectroscopy," Opt. Express 17, 1282-1290 (2009).

9. C. P. Pfeffer, B. R. Olsen, F. Ganikhanov, and F. Legare, "Multimodal nonlinear optical imaging of collagen arrays," J. Struct. Biol. 164, 140-145 (2008).

10. C. H. Chien, W. W. Chen, J. T. Wu, and T. C. Chang, "Label-free imaging of Drosophila in vivo by coherent anti-Stokes Raman scattering and two-photon excitation autofluorescence microscopy,” J. Biomed. Opt. 16, 016012 (2011).

11. J. Lin, F. Lu, W. Zheng, S. Xu, D. Tai, H. Yu, and Z. 
Huang, "Assessment of liver steatosis and fibrosis in rats using integrated coherent anti-Stokes Raman scattering and multiphoton imaging technique," J. Biomed. Opt. 16, 116024 (2011).

12. E. Belanger, J. Crepeau, S. Laffray, R. Vallee, Y. De Koninck, and D. Côté, "Live animal myelin histomorphometry of the spinal cord with video-rate multimodal nonlinear microendoscopy," J. Biomed. Opt. 17, 021107 (2012).

13. R. M. Hoffman, "Advantages of multi-color fluorescent proteins for whole-body and in vivo cellular imaging," J. Biomed. Opt. 10, 41202 (2005).

14. H. Kawano, T. Kogure, Y. Abe, H. Mizuno, and A. Miyawaki, "Two-photon dual-color imaging using fluorescent proteins," Nat. Methods 5, 373-374 (2008).

15. S. E. Tillo, T. E. Hughes, N. S. Makarov, A. Rebane, and M. Drobizhev, "A new approach to dual-color two-photon microscopy with fluorescent proteins," BMC Biotechnol. 10, 6 (2010).

16. P. Mahou, M. Zimmerley, K. Loulier, K. S. Matho, G. Labroille, X. Morin, W. Supatto, J. Livet, D. Debarre, and E. Beaurepaire, "Multicolor two-photon tissue imaging by wavelength mixing," Nat. Methods 9, 815-818 (2012).

17. S. Tang, T. B. Krasieva, Z. Chen, G. Tempea, and B. J. Tromberg, "Effect of pulse duration on two-photon excited fluorescence and second harmonic generation in nonlinear optical microscopy," J. Biomed. Opt. 11, 020501 (2006).

18. T. T. Le, I. M. Langohr, M. J. Locker, M. Sturek, and J. $\mathrm{X}$. Cheng, "Label-free molecular imaging of atherosclerotic lesions using multimodal nonlinear optical microscopy," J. Biomed. Opt. 12, 054007 (2007).

19. H. W. Wang, I. M. Langohr, M. Sturek, and J. X. Cheng, "Imaging and quantitative analysis of atherosclerotic lesions by CARS-based multimodal nonlinear optical microscopy," Arterioscler. Thromb. Vasc. Biol. 29, 1342-1348 (2009).

20. A. C. Ko, A. Ridsdale, M. S. Smith, L. B. Mostaco-Guidolin, M. D. Hewko, A. F. Pegoraro, E. K. Kohlenberg, B. Schattka, M. Shiomi, A. Stolow, and M. G. Sowa, "Multimodal nonlinear optical imaging of atherosclerotic plaque development in myocardial infarction-prone rabbits," J. Biomed. Opt. 15, 020501 (2010).

21. L. B. Mostaco-Guidolin, M. G. Sowa, A. Ridsdale, A. F. Pegoraro, M. S. Smith, M. D. Hewko, E. K. Kohlenberg, B. Schattka, M. Shiomi, A. Stolow, and A. C. Ko, "Differentiating atherosclerotic plaque burden in arterial tissues using femtosecond CARS-based multimodal nonlinear optical imaging," Biomed. Opt. Express 1, 59-73 (2010).

22. J. L. Suhalim, C.-Y. Chung, M. B. Lilledahl, R. S. Lim, M. Levi, B. J. Tromberg, and E. O. Potma, "Characterization of cholesterol crystals in atherosclerotic plaques using stimulated Raman scattering and second-harmonic generation microscopy," Biophys. J. 102, 1988-1995 (2012).

23. P. Libby, "Molecular bases of the acute coronary syndromes," Circulation 91, 2844-2850 (1995).

24. C. M. Dollery, J. R. McEwan, and A. M. Henney, "Matrix metalloproteinases and cardiovascular disease," Circ. Res. 77, 863-868 (1995).

25. J. L. Mehta, T. G. Saldeen, and K. Rand, "Interactive role of infection, inflammation and traditional risk factors in atherosclerosis and coronary artery disease," J. Am. Coll. Cardiol. 31, 1217-1225 (1998).

26. M. J. Davies, P. D. Richardson, N. Woolf, D. R. Katz, and J. Mann, "Risk of thrombosis in human atherosclerotic plaques: role of extracellular lipid, macrophage, and smooth muscle cell content," Br. Heart J. 69, 377-381 (1993).

27. G. Stoll and M. Bendszus, "Inflammation and atherosclerosis: Novel insights into plaque formation and destabilization," Stroke 37, 1923-1932 (2006).

28. M. M. Kockx, G. R. De Meyer, J. Muhring, W. Jacob, H. Bult, and A. G. Herman, "Apoptosis and related proteins in different stages of human atherosclerotic plaques," Circulation 97, 2307-2315 (1998).

29. D. J. Taatjes, M. P. Wadsworth, A. S. Quinn, J. H. Rand, E. G. Bovill, and B. E. Sobel, "Imaging aspects of cardiovascular disease at the cell and molecular level," Histochem. Cell Biol. 130, 235-245 (2008).

30. H. Azumi, K. Hirata, T. Ishida, Y. Kojima, Y. Rikitake, S. Takeuchi, N. Inoue, S. Kawashima, Y. Hayashi, H. Itoh, T. Quertermous, and M. Yokoyama, "Immunohistochemical localization of endothelial cell-derived lipase in atherosclerotic human coronary arteries," Cardiovasc. Res. 58, 647-654 (2003).

31. D. J. Schneider, M. Hayes, M. Wadsworth, H. Taatjes, M. Rincon, D. J. Taatjes, and B. E. Sobel, "Attenuation of neointimal vascular smooth muscle cellularity in atheroma by plasminogen activator inhibitor type 1 (PAI-1)," J. Histochem. Cytochem. 52, 1091-1099 (2004).

32. S.-H. Kim, E.-S. Lee, J. Lee, E. Lee, B.-S. Lee, J. Park, and D. Moon, "Multiplex coherent anti-Stokes Raman spectroscopy images intact atheromatous lesions and concomitantly identifies distinct chemical profiles of atherosclerotic lipids," Circ. Res. 106, 1332-1341 (2010).

33. W. Zipfel, "Two-photon excitation cross sections," http://www.drbio.cornell.edu/cross_sections.html.

34. O. de Barco and J. M. Bueno, "Second harmonic generation signal in collagen fibers: role of polarization, numerical aperture, and wavelength," J. Biomed. Opt. 17, 045005 (2012).

35. J. X. Rong, M. Shapiro, E. Trogan, and E. A. Fisher, “ Transdifferentiation of mouse aortic smooth muscle cells to a macrophage-like state after cholesterol loading," Proc. Natl. Acad. Sci. USA 100, 13531-13536 (2003).

36. P. Libby, P. M. Ridker, and A. Maseri, "Inflammation and atherosclerosis," Circulation 105, 1135-1143 (2002).

37. K. Jagavelu, U. J. Tietge, M. Gaestel, H. Drexler, B. Schieffer, and U. Bavendiek, "Systemic deficiency of the MAP kinase-activated protein kinase 2 reduces atherosclerosis in hypercholesterolemic mice," Circ. Res. 101, 1104-1112 (2007).

38. K. A. Campbell, M. J. Lipinski, A. C. Doran, M. D. Skaflen, V. Fuster, and C. A. McNamara, "Lymphocytes and the adventitial immune response in atherosclerosis," Circ. Res. 110, 889-900 (2012).

39. A. M. Kampoli, D. Tousoulis, C. Antoniades, G. Siasos, and C. Stefanadis, "Biomarkers of premature atherosclerosis," Trends Mol. Med. 15, 323-332 (2009). 